\title{
Metastability in specific heat measurements: simulations with the FPU model
}

\author{
A. Carati $^{1}$ and L. Galgani ${ }^{1}$ \\ 1 Department of Mathematics, University of Milano - Via Saldini 50, 20133 Milano, \\ Italy
}

PACS. 05.45.-a - First pacs description.

PACS. 05.20.-y - Second pacs description.

\begin{abstract}
In this letter we report numerical results giving, as a function of time, the energy fluctuation of a Fermi-Pasta-Ulam system in dynamical contact with a heat bath, the initial data of the FPU system being extracted from a Gibbs distribution at the same temperature of the bath. The aim is to get information on the specific heat of the FPU system in the spirit of the fluctuation-dissipation theorem. While the standard equilibrium result is recovered at high temperatures, there exists a critical temperature below which the energy fluctuation as a function of time tends to an asymptotic value sensibly lower than the one expected at equilibrium. This fact appears to exhibit the existence of a metastable state for generic initial conditions. An analogous phenomenon of metastability was up to now observed in FPU systems only for exceptional initial data having vanishing Gibbs measure, namely excitations of a few low-frequency modes (as in the original FPU work).
\end{abstract}

Introduction. - The Fermi-Pasta-Ulam system, i.e. a chain of particles interacting through (nearest-neighbour) weakly non-linear forces, was introduced (see [1]) with the aim of clarifying a fundamental problem in statistical mechanics, namely to understand how quickly is equilibrium attained (the so-called "problem of the rate of thermalization"). The state of the art can be summarized as follows. By numerical simulations with excitations of a few low-frequency modes, it was found that equipartition of the (time-averaged) mode energies, which is predicted by equilibrium statistical mechanics, is attained rather quickly if the initial energy is above a certain threshold. Below such a threshold one finds instead (see [2]), a quick relaxation to a state in which equipartition of energy obtains only within a packet of lowfrequency modes, with an exponential tail towards the high-frequencies. The time needed for the formation of such a packet is found to increase as an inverse power of the specific energy as the latter is diminished. The subsequent evolution to the final equilibrium state (with full equipartition) occurs instead on a much longer time scale, increasing as a stretched exponential with inverse specific energy. Semi-analytical and numerical indications were first given in [3] for the $\varphi^{4}$-model, and later confirmations for the FPU-model were given for example in [4]. So, at low enough temperatures the system behaves as if it had attained a final equilibrium, although it is actually being in a kind of metaequilibrium state, somehow analogous to the familiar ones of glasses. Reviews can be found in [5], [6] and [7].

(C) EDP Sciences 
Now, the set of initial data with excitations of a few low-frequency modes, which are the ones considered in the above-mentioned results, is statistically irrelevant in the thermodynamic limit, being exceptional with respect to the Gibbs measure. So there naturally arises the question whether an analogous phenomenon of metaequilibrium occurs also for typical initial data. On the other hand, typical initial data imply by definition equipartition of energy,

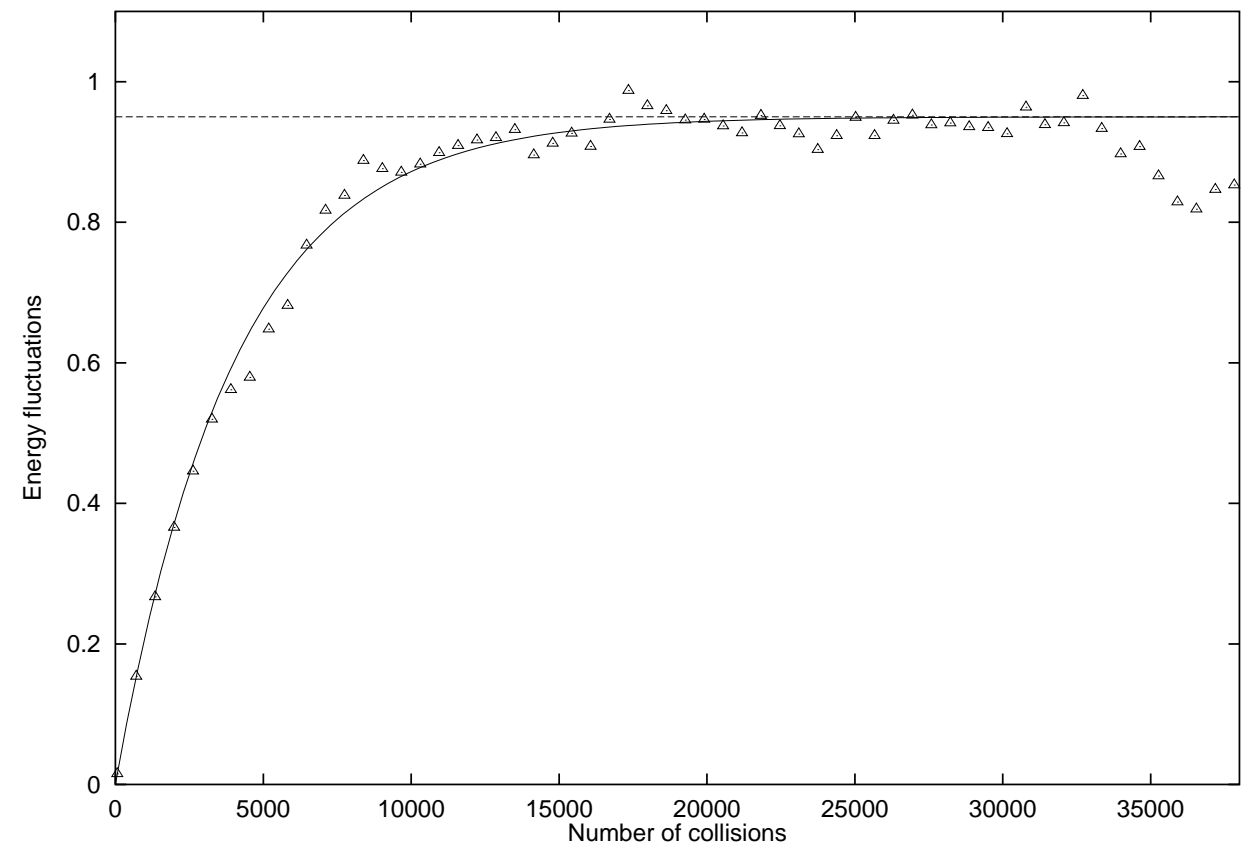

Fig. 1 - Energy fluctuation $F(n, T)$ versus number of collisions (in units of a thousand) at temperature $T=1$ for 100 particles. The fitting curve is discussed later in the text. The horizontal line gives the value 0.95 of the equilibrium specific heat at $T=1$, with the nonlinearity taken into account.

so that it not clear which quantity should be observed in order to test whether a metaequilibrium state rather than an equilibrium one is being attained. In the present paper we propose a concrete quantity related to specific heat measurements, which is suitable to this end. Moreover, we report the results of some numerical computations, which appear to indicate that metaequilibrium states in connection with specific heat measurements actually do show up in a FPU system for typical initial data at low enough temperatures.

To this end, we make reference to the formula relating equilibrium specific heat and temporal energy fluctuations according to the fluctuation-dissipation theorem, namely

$$
\lim _{t \rightarrow+\infty}<(E(t)-E(0))^{2}>_{T} /\left(2 N T^{2}\right)=C_{V}(T) .
$$

Here one considers a system in dynamical contact with a heat bath at a given temperature 
$T$, and $E(t)$ is the system's energy at time $t$; furthermore, $\left\langle\cdot>_{T}\right.$ denotes Gibbs average over the initial data at the same temperature $T$, while $C_{V}(T)$ is the corresponding equilibrium (canonical) specific heat (with the Boltzmann constant $k_{B}=1$ ).

The quantity that we propose as a suitable observable is then nothing but the function

$$
F(t, T) \stackrel{\text { def }}{=}<(E(t)-E(0))^{2}>_{T} /\left(2 N T^{2}\right)
$$

itself. One should compute it for an FPU system in dynamical contact with a heat bath at temperature $T$, and plot it versus time. Indeed, on the one hand, such a quantity by definition makes reference to typical initial data. On the other hand, when plotted as a function of time, it starts up from zero and should attain, after a certain relaxation time, the equilibrium value $C_{V}(T)$. The relaxation time can then be concretely estimated as a function of temperature $T$. Moreover, one might agree that a metaequilibrium state has been exhibited if, for a given temperature $T$, the quantity $F(t, T)$ as a function of time is found to have relaxed to some definite value, sensibly lower than the equilibrium one. Indeed, in such a case, that value should legitimately be considered as estimating the specific heat which is observed in an actual measurement of time-length $t$.

The model of interaction of the FPU system with a heat bath. - The FPU system we consider is a chain of $N+2$ particles of equal mass $m$ with fixed ends, interacting through a first neighbour quartic potential (the so called $\beta$-model), i.e., with Hamiltonian

$$
H_{\mathrm{F}}(p, q)=\sum_{i=1}^{N} \frac{p_{i}^{2}}{2 m}+\sum_{i=0}^{N}\left[\frac{\Omega^{2}}{2}\left(q_{i}-q_{i+1}\right)^{2}+\frac{\beta}{4}\left(q_{i}-q_{i+1}\right)^{4}\right],
$$

with boundary conditions $q_{0}=q_{N+1}=0$, while $\Omega$ and $\beta$ are suitable parameters.

The interaction with a heat bath is modeled as follows. In principle, we think of the heat bath as a perfect gas constituted by a very large number of particles, each of which interacts, via a suitable rapidly decreasing potential, with the leftmost moving particle of the FPU system. Each collision then produces a certain exchange of energy between the body and the heat bath. This is indeed the model we actually implemented in a similar context (see [8]). However, in the present context, a very large number of particles would be needed for an appropriate simulation of the heat bath, and this is highly impracticable. Thus, we chose as a model the related one in which the collisions with all the molecules of a large bath are replaced by the successive collisions with a single gas particle, the initial data of the particle before each collision being extracted from a Maxwell distribution at temperature $T$; further details are given later. Denoting by $x, p_{x}$ the position and the momentum of the incoming gas particle, we thus consider the totat system with Hamiltonian

$$
H\left(p_{x}, x, p, q\right)=p_{x}^{2} / 2 m+V\left(x-q_{1}\right)+H_{\mathrm{F}}(p, q) .
$$

The interaction potential $V$ is taken of the form $V(r)=V_{0} \cdot(L / r) \exp (-r / L)$, where $V_{0}$ and $L$ are the strength and the range respectively of the potential. The quantities $m, V_{0}$ and $L$ are chosen as units of mass, energy and length respectively, while the parameters $\Omega$ and $\beta$ are determined by a power expansion about the equilibrium point of a Lennard-Jones potential having $V_{0}$ as depth and $L$ as range. In this way, the unusual values $\Omega=20$ and $\beta=3600$ are obtained.

Concerning the initial data of the gas particle, for the position we take $\left|x_{o}\right|=20 \mathrm{~L}$ (so that the initial interaction potential is negligible), while the momentum $p_{x o}$ is extracted at random according to a Maxwell distribution at a given temperature $T$. More precisely, it is 


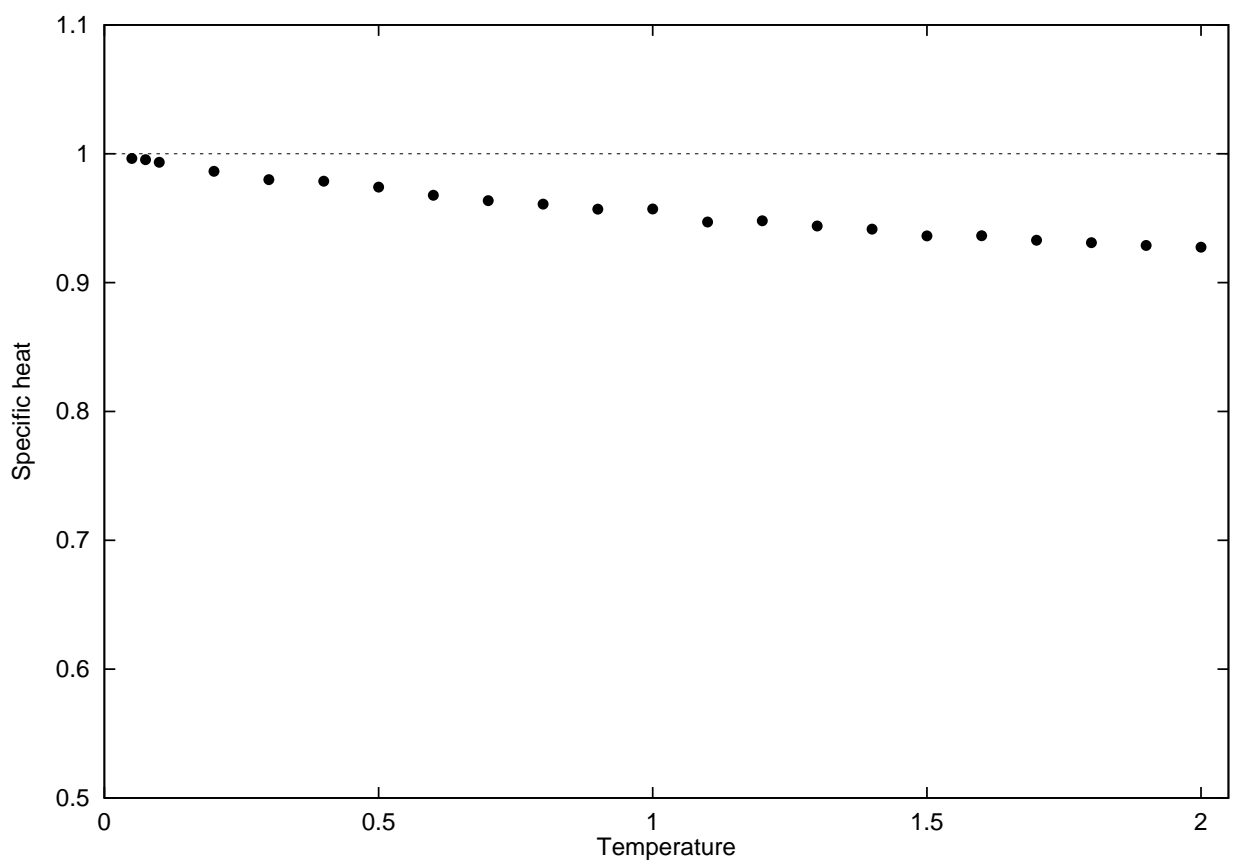

Fig. 2 - Specific heat versus temperature, with the anharmonicity taken into account. The corresponding harmonic value 1 is also indicated.

well known that the given constraint on the initial position requires a correction factor for the Maxwellian: in fact, the velocity has to be extracted from a distribution with density $\rho\left(p_{x}\right)=C p_{x} \exp \left(-p_{x}^{2} / 2 m T\right)$, with a normalization constant $C$. Concerning the initial data for the FPU system, they are extracted from a Gibbs distribution at temperature $T$, with reference to the full anharmonic Hamiltonian $H_{\mathrm{F}}$ involving the quartic terms. The technical way in which this was implemented is described later.

Preliminary results; attainment of equilibrium at high temperatures. - Having thus chosen the initial data in the said way, the equations of motion corresponding to the total Hamiltonian $H$ are integrated by the leap-frog method, and the collision is considered to have terminated when the position of the gas particle becomes again equal to $x_{o}$. At such a moment we read the value $E$ of the energy of the FPU system. We then iterate the procedure by extracting each time a new random velocity for the incoming particle, while the data for the FPU system are left unchanged, i.e. the initial FPU data for the new collision are just equal to the final ones of the previous collision. Thus one obtains a sequence $\left\{E_{n}\right\}$ of energy values of the FPU system, and the exchanged energy up to "time" $n$ is then $(\Delta E)_{n} \stackrel{\text { def }}{=} E_{n}-E_{0}$, where $E_{0}$ is the initial energy. In order to estimate the quantity $F(n, T)=<\left(E_{n}-E_{0}\right)^{2}>_{T}$ $/\left(2 N T^{2}\right)$ which appears in $(2)$, one has to repeat the above procedure a sufficient number 


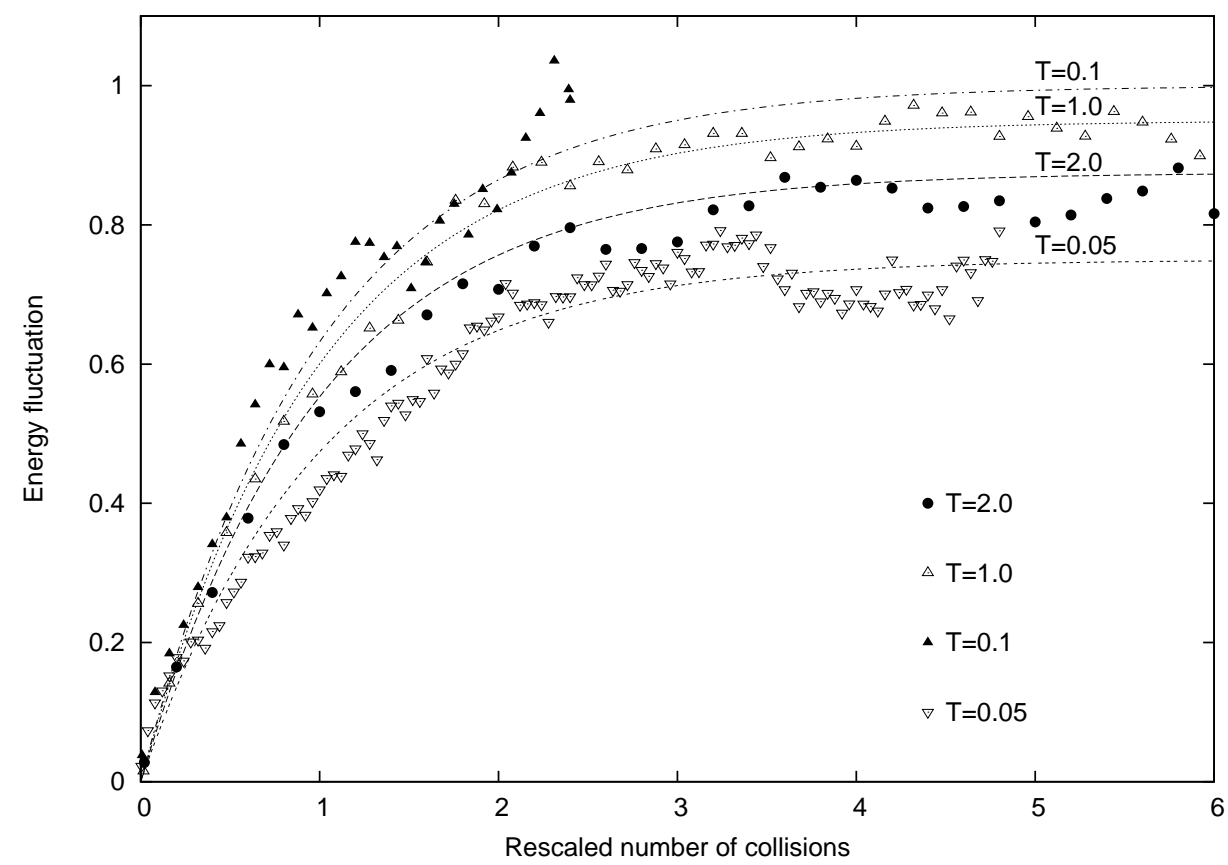

Fig. 3 - Energy fluctuation versus rescaled number of collisions, with the corresponding fits. The simulation for $T=0.05$ took a one month work of a parallel machine using 52 CPUs.

$K$ of times, each time choosing the initial data for the FPU system at random from a Gibbs distribution at temperature $T$. Thus, one has $K$ initial energies $E_{0}^{i}, i=1, \ldots, K$, and $K$ energy sequences $\left\{E_{n}^{i}\right\}$, each one corresponding to $n$ collisions, and finally one sets $<\left(E_{n}-E_{0}\right)^{2}>_{T}=$ $(1 / K) \sum_{i=1}^{K}\left(E_{n}^{i}-E_{0}^{i}\right)^{2}$.

An example of the results obtained in such a way is illustrated in Figure 1, where the quantity $<\left(E_{n}-E_{0}\right)^{2}>_{T} /\left(2 N T^{2}\right)$, which from now on will be called the "energy fluctuation" (but might rather be called the mean squared "energy jump") is plotted versus the number $n$ of collisions. The figure corresponds to the case of a FPU system with $N=100$ moving particles, at temperature $T=1$ (in our units), with $K=640$. One sees that the energy fluctuation starts up growing from 0 , and after a number $n \simeq 3 \cdot 10^{4}$ of collisions appears to have attained an asymptotic value $\simeq 0.95$. We recall that, for a purely quadratic FPU Hamiltonian, the equilibrium value of $C_{V}(T)$ is independent of temperature and equal to 1 . However, in order to check whether the observed "final value" agrees with the predictions of equilibrium statistical mechanics or not, the anharmonic contribution has to be taken into account.

In order to illustrate how this was performed, we start describing how the anharmonic contribution was taken into account in the related problem of extracting the initial data 
according to the Gibbs distribution proportional to $\exp \left(-H_{\mathrm{F}}(p, q) / T\right)$. To this end we make use of the so called rejection method [9]. Namely, we start considering only the quadratic part (say $H_{2}(p, q)$ ) of the FPU Hamiltonian $H_{\mathrm{F}}(p, q)$, because in such a case the normal modes are distributed as independent gaussian variables. Having extracted the values for the normal modes according to the harmonic Hamiltonian, we then read the numbers $E_{0}=H_{\mathrm{F}}(p, q)$ and $\hat{E}_{0}=H_{2}(p, q)$. The values are rejected if, having chosen at random a value $z$ in $(0,1)$, one has $z>\exp \left(\left(\hat{E}_{0}-E_{0}\right) / T\right)$, while they are accepted in the opposite case. The sequence of accepted values is known to be distributed according to a density proportional to $\exp \left(-H_{\mathrm{F}}(p, q) / T\right)$. In this way we are also able to numerically evaluate the canonical specific heat for the full Hamiltonian $H_{\mathrm{F}}$, by just evaluating at each temperature $T$ the standard deviation of the distribution and using the familiar canonical formula relating standard deviation and specific heat.

The results of such a procedure are reported in Figure 2, where the equilibrium (canonical) specific heat $C_{V}$ for the full Hamiltonian $H_{\mathrm{F}}$ is plotted versus temperature $T$. The computations were performed by extracting a sample of $K=10^{6}$ data at each temperature, and using the formula $N T^{2} C_{V}=\sum_{i=1}^{K}\left(E_{0}^{i}\right)^{2} / K-\left(\sum_{i=1}^{K} E_{0}^{i} / K\right)^{2}$. From Figure 2, one sees that at temperature $T=1$ one has for the equilibrium specific heat a value $\simeq 0.95$, which is in very good agreement with the asymptotic value of the dynamical energy fluctuation reported in Figure 1. We conclude that at temperature $T=1$ relation (1) is well satisfied and that the corresponding relaxation time is shorter than the available computation time.

Metastability at a low temperature. - Now we can proceed to compute the energy fluctuation as a function of time for other values of temperature $T$, in order to check whether an asymptotic values is actually attained and whether the latter agrees with the final one expected according to (1). The first result we find is that the number of collisions needed to attain an asymptotic regime increases substantially as temperature is lowered. This is expected, since the mean velocity of the gas particle decreases, which entails smaller energy exchanges (see for example [10] for the case of diatomic molecules). The increase of the relaxation time actually turns out to be so steep, that we cannot afford to observe a relaxation at all in simulations with $T<0.05$, and even for $T=0.05$ it took a month to observe a relaxation with a computer using 52 parallel processors.

From several simulations at $T>0.05$ we found that the number of collisions needed to observe a relaxation scales as $1 / T^{2}$. This was obtained by fitting the family of curves $F(n, T) \stackrel{\text { def }}{=}<\left(E_{n}-E_{0}\right)^{2}>_{T} /\left(2 N T^{2}\right)$ to curves of the form

$$
F(n, T)=A_{\infty}(T)\left(1-\exp \left(-n / n_{0}(T)\right),\right.
$$

which gave $n_{0}(T)=4 \cdot 10^{3} / T^{2}$.

Our main result is reported in Figure 3, where the energy fluctuation $F(n, T)$ is plotted versus the rescaled number of collisions $n / n_{0}(T)$, for different temperatures; the above mentioned interpolations are also reported. First we considered a temperature larger than $T=1$, namely $T=2$. The expected asymptotic value is now smaller, namely $\simeq 0.92$, due to the increased anharmonic contribution (which has a negative sign) to the specific heat. The energy fluctuation appears indeed to have relaxed to a smaller value than at $T=1$, and actually the best fit gives $A_{\infty}=0.87$, so that there is at least a qualitative agreement. The relevant step however is now to go to lower temperatures, where qualitative discrepancies with respect to equilibrium statistical mechanics may occur. Notice by the way that the anharmonic contribution to the specific heat steadily decreases with decreasing temperature, so that the equilibrium value steadily approaches the harmonic value 1 , and is essentially indistinguish- 
able from it for example at $T=0.1$. This fact is actually observed also for the corresponding dynamical quantity at temperature $T=0.1$, as the results of Figure 3 show. One will notice that the computations were performed in such a case only for a rather short time. The reason was that the computer times become formidable with decreasing temperatures. So we decided that the indications available at $T=0.1$ were sufficient, and concentrated our attention to the case $T=0.05$, with a run that, as mentioned previously, took one month of computation. The results were however quite rewarding. Indeed, here too a rather good approach to some asymptotic value is obtained. But there is however an inversion in the trend of the asymptotic value because the present one, instead of being still nearer to 1, turns out to have sensibly diminished, being $\simeq 0.75$.

We interpret this result as a strong indication that a metastable state has been attained. Indeed here too, in analogy with the case of initial excitations of a few low-frequency modes, we may expect that the energy fluctuation will eventually attain the equilibrium canonical limit. But the results of Figure 3 suggest that at $T=0.05$ the final relaxation would require a number of collisions much larger than $n_{0}(T)$. In other terms, the time-scale to the final equilibrium is expected to be much larger than the one leading to the relaxation observed here. Such an occurring of (at least) two different time-scales indeed is a characteristic feature of metastability phenomena. Our numerical results thus appear to be a direct indication that, below a certain critical temperature ( $T=0.05$ in our units), two time-scales, and thus a metastability phenomenon, do show up for initial data of full measure, in connection with measurements of the specific heat. For a previous indication, and the suggestion of an analogy with the phenomenon of the glass transition, see [8].

Discussion. - The conception that the measured values of the specific heat of a system may depend on the observation time is today a currently accepted one, at least for a certain class of materials such as supercooled liquids near the glass transition or amorphous material. For example, one finds papers (see [11]) by the title "Observation of time-dependent specific heat in amorphous $\mathrm{SiO}_{2}$ ". A very good illustration of the situation is given in the paper Birge [12] (see also [13]), where one even finds the statement: "Clearly the dynamics of how one performs the measurement enters into the measured value". In any case, the attention is addressed to the fact that one is dealing with situations in which "the system under observation falls out of complete thermodynamic equilibrium at some point of the experiment" (see [14]). Thus, "The problem with studying static thermodynamic quantities such as $c_{p}$ is that their significance changes at the temperature $T_{g}$ where the system falls out of equilibrium. How does one interpret a quantity such as $c_{p}$ in a nonequilibrium situation?".

So, the results reported in the present paper appear to indicate that at least some part of the phenomenology of the time-dependent specific heats observed in the supercooled liquids near the glass transition do occur also in numerical simulations of specific heat measurement for FPU systems. Perhaps, this indication can also be considered as suggesting that an analogous phenomenonology may be observed in actual specific heat measuremets of crystals near the Debye temperature.

In this connection, it may be of interest to recall that some relevant indications to this effect are known already since the work of Nernst [15] of the year 1911 (see also [16]), where for the first time a systematic study was performed of the specific heats of crystals at low temperatures, and some anomaly was observed concerning the times needed for the stabilization of the specific heat measurements. Indeed Nernst was struck by the fact that the stabilization times were found to increase so much as temperature was decreased, as to even suggest that the implementation itself of the measurements may be impossible at small temperatures. Instead, quite unexpectedly the times were found to present a decrease of several 
orders of magnitude just at the temperatures at which the specific heat did stabilize to values sensibly smaller than the "classical ones" (see also [17]). We hope to have the opportunity to perform further investigations in order to ascertain whether an analogous phenomenon may be observed in numerical simulations on FPU models.

\section{REFERENCES}

[1] Fermi E., Pasta J. and Ulam S., in Fermi E., Collected Papers (University of Chicago Press, Chicago) 1965.

[2] Berchialla L., Galgani L. and Giorgilli A., DCDS A, 11 (2004) 855.

[3] Fucito E., Marchesoni F., Marinari E., Parisi G., Peliti L., Ruffo S. and Vulpiani A., Journ. de Phys., 43 (1982) 707 PARISI G., Europhysics Lett., 40 (1997) 357.

[4] Berchialla L., Giorgilli A. and Paleari S., Phys. Lett. A, 321 (2004) 167.

[5] Carati A., Galgani L., Giorgilli A. and Ponno A., Nuovo Cim. B, 117 (2002) 1017.

[6] Carati A., Galgani L. and Giorgilli, Chaos, 15 (2005) 015107.

[7] Benettin G., Carati A., Galgani L. and Giorgilli A., Conference on the FPU problem, edited by Gallavotti G. (Springer, Berlin) 2006.

[8] Carati A. and Galgani L., J. Stat. Phys., 94 (1999) 859.

[9] Devroy L., Non-Uniform Random Variate Generation (Springer-Verlag, New York) 1986.

[10] Carati A., Galgani L. and Pozzi B., Phys. Rev. Lett., 90 (2003) 010601.

[11] Loponen M.T., Dynes R.C., Naraynamurti V. and Garno J.P., Phys. Rev. Lett., 45 (1980) 457.

[12] Birge N. O., Phys. Rev. B, 34 (1986) 1631.

[13] Birge N. O. and NaAgel S.R., Phys. Rev. Lett. B, 25 (1985) 2674.

[14] Angell C. A. and Sichina W., Ann. N. Y. Acad. Sci., 279 (1976) 53.

[15] Nernst W., Annalen d. Physik, 36 (1911) 395

[16] Gunther P., Annalen d. Physik, 51 (1916) 828.

[17] Fluchaber P., Leadbetter A.J. and Morrison J.A., Proc. Philos. Soc. (London), 78 (1961) 1449. 